\title{
Estimation of Reliability in Multicomponent Stress-Strength Based on Generalized Rayleigh Distribution
}

Gadde Srinivasa Rao

University of Dodoma, Dodoma, Tanzania, gaddesrao@yahoo.com

Follow this and additional works at: http://digitalcommons.wayne.edu/jmasm

Part of the Applied Statistics Commons, Social and Behavioral Sciences Commons, and the Statistical Theory Commons

\section{Recommended Citation}

Rao, Gadde Srinivasa (2014) "Estimation of Reliability in Multicomponent Stress-Strength Based on Generalized Rayleigh Distribution," Journal of Modern Applied Statistical Methods: Vol. 13 : Iss. 1, Article 24.

DOI: $10.22237 /$ jmasm/1398918180

Available at: http://digitalcommons.wayne.edu/jmasm/vol13/iss1/24 


\title{
Estimation of Reliability in Multicomponent Stress-Strength Based on Generalized Rayleigh Distribution
}

\author{
Gadde Srinivasa Rao \\ University of Dodoma \\ Dodoma, Tanzania
}

A multicomponent system of $k$ components having strengths following $k$ - independently and identically distributed random variables $x_{1}, x_{2}, \ldots, x_{k}$ and each component experiencing a random stress $Y$ is considered. The system is regarded as alive only if at least $s$ out of $k(s<k)$ strengths exceed the stress. The reliability of such a system is obtained when strength and stress variates are given by a generalized Rayleigh distribution with different shape parameters. Reliability is estimated using the maximum likelihood (ML) method of estimation in samples drawn from strength and stress distributions; the reliability estimators are compared asymptotically. Monte-Carlo simulation is used to compare reliability estimates for the small samples and real data sets illustrate the procedure.

Keywords: Generalized Rayleigh distribution, reliability estimation, stress-strength, ML estimation, confidence intervals

\section{Introduction}

Surles and Padgett $(1998,2001)$ introduced the two-parameter Burr Type X distribution and named it the generalized Rayleigh distribution. Note that the twoparameter generalized Rayleigh distribution is a particular member of the generalized Weibull distribution, originally proposed by Mudholkar and Srivastava (1993). The two-parameter Burr Type X distribution is referred to as the generalized Rayleigh distribution (GRD). For $\alpha>0$ and $\lambda>0$, the twoparameter GRD has the density function;

$$
f(x ; \alpha, \lambda)=2 \alpha \lambda^{2} x e^{-(\lambda x)^{2}}\left(1-e^{-(\lambda x)^{2}}\right)^{\alpha-1} \text { for } x>0
$$

Dr. G. Srinivasa Rao is a Professor of Statistics. Email at: gaddesrao@yahoo.com. 


\section{ESTIMATION OF RELIABILITY IN STRESS-STRENGTH}

and the distribution function is given by

$$
F(x ; \alpha, \lambda)=\left(1-e^{-(\lambda x)^{2}}\right)^{\alpha} \quad \text { for } x>0
$$

Here $\alpha$ and $\lambda$ are the shape and scale parameters respectively. The GRD has been studied extensively by Kundu and Raqab (2005) and Raqab and Kundu (2005). The two-parameter GRD is denoted by $\operatorname{GR}(\alpha, \lambda)$. Surles and Padgett (2001) showed that the two-parameter GR distribution can be used effectively in modeling strength as well as general lifetime data.

This article studies reliability in a multicomponent stress-strength based on $X, Y$, two independent random variables, where $X$ and $Y$ fallow generalized Rayleigh distributions with shape parameters $\alpha$ and $\beta$ respectively and with common scale parameter $\lambda$.

Let the random samples $y, x_{1}, x_{2}, \ldots x_{k}$ be independent, $G(y)$ be the continuous distribution function of $Y$ and $F(x)$ be the common continuous distribution function of $x_{1}, x_{2}, \ldots x_{k}$. The reliability in a multicomponent stressstrength model developed by Bhattacharyya and Johnson (1974) is

$$
\begin{aligned}
R_{s, k} & =\mathrm{P}\left[\text { at least } s \text { of the }\left(x_{1}, x_{2}, \ldots x_{k}\right) \text { exceed } Y\right] \\
& =\sum_{i=s}^{k}\left(\begin{array}{c}
k \\
i
\end{array}\right) \int_{-\infty}^{\infty}[1-G(y)]^{i}[G(y)]^{k-i} d F(y),
\end{aligned}
$$

where $x_{1}, x_{2}, \ldots x_{k}$ are independently and identically distributed (iid) with common distribution function $F(x)$, this system is subjected to common random stress $Y$. The probability in (3) is called reliability in a multicomponent stress-strength model (Bhattacharyya \& Johnson, 1974). The survival probability of single component stress-strength versions have been considered by several authors assuming various lifetime distributions for the stress-strength random variates (Enis \& Geisser, 1971; Downtown, 1973; Awad \& Gharraf, 1986; McCool, 1991; Nandi \& Aich, 1994; Surles \& Padgett, 1998; Raqab \& Kundu, 2005; Kundu \& Gupta, 2005, 2006; Raqab, et al., 2008; Kundu \& Raqab, 2009). Reliability in a multicomponent stress-strength was developed by Bhattacharyya and Johnson (1974) and Pandey and Borhan Uddin (1985) and the references therein cover the study of estimating $P(Y<X)$ in many standard distributions assigned to one or both of stress, strength variates. Recently Srinivasa Rao and Kantam (2010) 


\section{GADDE SRINIVASA RAO}

studied estimation of reliability in multicomponent stress-strength for log-logistic distribution.

Suppose a system, with $k$ identical components, functions if $s(1 \leq s \leq k)$ or more of the components simultaneously operate. In its operating environment, the system is subjected to a stress $Y$ which is a random variable with distribution function $G($.$) . The strengths of the components, that is the minimum stresses to$ cause failure, are independent and identically distributed random variables with distribution function $F($.$) . Then the system reliability, which is the probability that$ the system does not fail, is the function $R_{s, k}$ given in (3). The estimation of survival probability in a multicomponent stress-strength system when the stress, strength variates are following Rayleigh distribution is not paid much attention. Therefore, this article studies the estimation of reliability in multicomponent stress-strength model with reference to Rayleigh distribution.

\section{Maximum Likelihood Estimator of $R_{s, k}$}

Let $X \sim G R(\alpha, \lambda)$ and $Y \sim G R(\beta, \lambda)$ with unknown shape parameters $\alpha, \beta$ and common scale parameter $\lambda$, where $X$ and $Y$ are independently distributed. The reliability in multicomponent stress- strength for generalized Rayleigh distribution using (3) results in:

$$
\begin{aligned}
R_{s, k} & =\sum_{i=s}^{k}\left(\begin{array}{c}
k \\
i
\end{array}\right) \int_{0}^{\infty}\left[1-\left(1-e^{-(\lambda y)^{2}}\right)^{\beta}\right]^{i}\left[\left(1-e^{-(\lambda y)^{2}}\right)^{\beta}\right]^{k-i} 2 \alpha \lambda^{2} y e^{-(\lambda y)^{2}}\left(1-e^{-(\lambda y)^{2}}\right)^{\alpha-1} d y \\
& =\sum_{i=s}^{k}\left(\begin{array}{c}
k \\
i
\end{array}\right) \int_{0}^{1}\left[1-t^{\beta}\right]^{i}\left[t^{\beta}\right]^{k-i} \alpha t^{\alpha-1} d t \quad \text { where } t=1-e^{-(\lambda y)^{2}} \\
& =\sum_{i=s}^{k}\left(\begin{array}{c}
k \\
i
\end{array}\right) v \int_{0}^{1} z^{k-i+v-1}[1-z]^{i} d z \quad \text { if } z=t^{\beta}, v=\frac{\alpha}{\beta} \\
& =v \sum_{i=s}^{k}\left(\begin{array}{c}
k \\
i
\end{array}\right) \beta(k+v-i, i+1) .
\end{aligned}
$$

After simplification this reduces to

$$
R_{s, k}=v \sum_{i=s}^{k} \frac{k !}{(k-i) !}\left[\prod_{j=0}^{i}(k+v-j)\right]^{-1}
$$




\section{ESTIMATION OF RELIABILITY IN STRESS-STRENGTH}

because $k$ and $i$ are integers. The probability in (4) is called reliability in a multicomponent stress-strength model. If $\alpha$ and $\beta$ are not known, it is necessary to estimate $\alpha$ and $\beta$ to estimate $R_{s, k}$. In this article $\alpha$ and $\beta$ are estimated using the ML method. The estimates are substituted in $v$ to obtain an estimate of $R_{s, k}$ using equation (4).

It is known that the method of Maximum Likelihood Estimation (MLE) has invariance property. In this direction, this article proposes the ML estimator for the reliability of a multicomponent stress-strength model by considering the estimators of the parameters of stress, strength distributions by ML method of estimation in a generalized Rayleigh distribution.

Let $x_{1}<x_{2}<\ldots .<x_{n} ; y_{1}<y_{2}<\ldots .<y_{m}$ be two ordered random samples of size $n, m$ respectively on strength, stress variates each following GRD with shape parameters $\alpha$ and $\beta$, common scale parameter $\lambda$. The log-likelihood function of the observed sample is

$$
\begin{aligned}
L(\alpha, \beta, \lambda)= & (n+m) \ln 2+n \ln \alpha+m \ln \beta \\
& +2(n+m) \ln \lambda-\lambda^{2}\left[\sum_{i=1}^{n} x_{i}^{2}+\sum_{j=1}^{m} y_{j}^{2}\right]+\sum_{i=1}^{n} \ln x_{i}+\sum_{j=1}^{m} \ln y_{j} \\
& +(\alpha-1) \sum_{i=1}^{n} \ln \left(1-e^{-\left(\lambda x_{i}\right)^{2}}\right)+(\beta-1) \sum_{j=1}^{m} \ln \left(1-e^{-\left(\lambda y_{j}\right)^{2}}\right)
\end{aligned}
$$

The MLEs of $\alpha, \beta$ and $\lambda$, for example, $\hat{\alpha}, \hat{\beta}$ and $\hat{\lambda}$, respectively can be obtained as the iterative solution of

$$
\begin{aligned}
& \frac{\partial \mathrm{L}}{\partial \alpha}=0 \Rightarrow \frac{n}{\alpha}+\sum_{i=1}^{n} \ln \left(1-e^{-\left(\lambda x_{i}\right)^{2}}\right)=0 \\
& \frac{\partial \mathrm{L}}{\partial \beta}=0 \Rightarrow \frac{m}{\beta}+\sum_{j=1}^{m} \ln \left(1-e^{-\left(\lambda y_{j}\right)^{2}}\right)=0
\end{aligned}
$$




\section{GADDE SRINIVASA RAO}

$$
\begin{aligned}
\frac{\partial \mathrm{L}}{\partial \lambda} & =0 \Rightarrow \frac{2(m+n)}{\lambda}-2 \lambda\left[\sum_{i=1}^{n} x_{i}^{2}+\sum_{j=1}^{m} y_{j}^{2}\right]+(\alpha-1) \sum_{i=1}^{n} \frac{2 \lambda x_{i}^{2} e^{-\left(\lambda x_{i}\right)^{2}}}{1-e^{-\left(\lambda x_{i}\right)^{2}}} \\
& +(\beta-1) \sum_{j=1}^{m} \frac{2 \lambda y_{j}^{2} e^{-\left(\lambda y_{j}\right)^{2}}}{1-e^{-\lambda y_{j}}}=0
\end{aligned}
$$

and then from (6), (7) and (8)

$$
\begin{aligned}
& \hat{\alpha}=\frac{-n}{\sum_{i=1}^{n} \ln \left(1-e^{-\left(\hat{\lambda} x_{i}\right)^{2}}\right)} \\
& \hat{\beta}=\frac{-m}{\sum_{j=1}^{m} \ln \left(1-e^{-\left(\hat{\lambda} y_{j}\right)^{2}}\right)}
\end{aligned}
$$

where $\hat{\lambda}$ can be obtained as the solution of non-linear equation

$$
\begin{aligned}
g(\lambda)= & \Rightarrow \\
& \frac{m+n}{\lambda}-\frac{n \lambda \sum_{i=1}^{n} \frac{x_{i}^{2} e^{-\left(\lambda x_{i}\right)^{2}}}{1-e^{-\left(\lambda x_{i}\right)^{2}}}}{\sum_{k=1}^{n} \ln \left(1-e^{-\left(\lambda x_{k}\right)^{2}}\right)}-\frac{m \sum_{j=1}^{m} \frac{y_{j}^{2} e^{-\left(\lambda y_{j}\right)^{2}}}{1-e^{-\left(\lambda y_{j}\right)^{2}}}}{\sum_{k=1}^{m} \ln \left(1-e^{-\left(\lambda y_{k}\right)^{2}}\right)} \\
& -\sum_{i=1}^{n} \frac{\lambda x_{i}^{2}}{1-e^{-\left(\lambda x_{i}\right)^{2}}}-\sum_{j=1}^{m} \frac{\lambda y_{j}^{2}}{1-e^{-\left(\lambda y_{j}\right)^{2}}}=0
\end{aligned}
$$

Therefore, $\hat{\lambda}$ is simple iterative solution of non-linear equation $g(\lambda)=0$. Once $\hat{\lambda}$ is known, $\hat{\alpha}$ and $\hat{\beta}$ can be obtained from (9) and (10) respectively. Therefore, the MLE of $R_{s, k}$ becomes

$$
R_{s, k}=\hat{v} \sum_{i=s}^{k} \frac{k !}{(k-i) !}\left[\prod_{j=0}^{i}(k+\hat{v}-j)\right]^{-1} \text { where } \hat{v}=\frac{\hat{\alpha}}{\hat{\beta}} .
$$




\section{ESTIMATION OF RELIABILITY IN STRESS-STRENGTH}

The asymptotic confidence interval for $R_{s, k}$, is calculated as: First, the asymptotic variance of the MLE is given by

$$
\mathrm{V}(\hat{\alpha})=\left[E\left(-\frac{\partial^{2} L}{\partial \alpha^{2}}\right)\right]^{-1}=\frac{\alpha^{2}}{n} \text { and } \mathrm{V}(\hat{\beta})=\left[E\left(-\frac{\partial^{2} L}{\partial \beta^{2}}\right)\right]^{-1}=\frac{\beta^{2}}{m}
$$

The asymptotic variance (AV) of an estimate of $R_{s, k}$ which a function of two independent statistics, for example, $\alpha, \beta$ is given by Rao (1973).

$$
\mathrm{AV}\left(\hat{\mathrm{R}}_{s, k}\right)=\mathrm{V}(\hat{\alpha})\left(\frac{\partial \mathrm{R}_{s, k}}{\partial \alpha}\right)^{2}+\mathrm{V}(\hat{\beta})\left(\frac{\partial \mathrm{R}_{s, k}}{\partial \beta}\right)^{2}
$$

From the asymptotic optimum properties of MLEs (Kendall \& Stuart, 1979) and of linear unbiased estimators (David, 1981), it is known that MLEs are asymptotically equally efficient having the Cramer-Rao lower bound as their asymptotic variance as given in (13). Thus, from Equation (14), the asymptotic variance of $\hat{R}_{s, k}$ can be obtained.

To avoid the difficulty of derivation of $R_{s, k}$, the derivatives of $R_{s, k}$ are obtained for $(s, k)=(1,3)$ and $(2,4)$ separately, they are given by

$$
\begin{aligned}
& \frac{\partial R_{1,3}}{\partial \alpha}=\frac{-3}{\beta(3+\hat{v})^{2}} \text { and } \frac{\partial R_{1,3}}{\partial \beta}=\frac{3 \hat{v}}{\beta(3+\hat{v})^{2}} \\
& \frac{\partial R_{2,4}}{\partial \alpha}=\frac{-12(7+2 \hat{v})}{\beta[(3+\hat{v})(4+\hat{v})]^{2}} \text { and } \frac{\partial R_{2,4}}{\partial \beta}=\frac{12 \hat{v}(7+2 \hat{v})}{\beta[(3+\hat{v})(4+\hat{v})]^{2}}
\end{aligned}
$$

Thus, $\operatorname{AV}\left(\hat{R}_{1,3}\right)=\frac{9 \hat{v}^{2}}{(3+\hat{v})^{4}}\left(\frac{1}{n}+\frac{1}{m}\right)$ and

$$
\begin{aligned}
& \operatorname{AV}\left(\hat{R}_{2,4}\right)=\frac{144 \hat{v}^{2}(7+2 \hat{v})^{2}}{[(3+\hat{v})(4+\hat{v})]^{4}}\left(\frac{1}{n}+\frac{1}{m}\right) . \\
& \text { As } n \rightarrow \infty, m \rightarrow \infty, \frac{\hat{R}_{s, k}-R_{s, k}}{\operatorname{AV}\left(\hat{R}_{s, k}\right)} \stackrel{d}{\longrightarrow} N(0,1),
\end{aligned}
$$




\section{GADDE SRINIVASA RAO}

and the asymptotic $100(1-\alpha) \%$ confidence interval for $R_{s, k}$ is given by

$$
\hat{R}_{s, k} \mp Z_{(1-\alpha / 2)} \sqrt{\operatorname{AV}\left(\hat{R}_{s, k}\right)}
$$

The asymptotic $100(1-\alpha) \%$ confidence interval for $R_{1,3}$ is given by

$$
\hat{R}_{1,3} \mp Z_{(1-\alpha / 2)} \frac{3 \hat{v}}{(3+\hat{v})^{2}} \sqrt{\left(\frac{1}{n}+\frac{1}{m}\right)} \text {, where } \hat{v}=\hat{\alpha} / \hat{\beta} \text {. }
$$

The asymptotic $100(1-\alpha) \%$ confidence interval for $R_{2,4}$ is given by

$$
\hat{R}_{2,4} \mp Z_{(1-\alpha / 2)} \frac{12 \hat{v}(7+2 \hat{v})}{[(3+\hat{v})(4+\hat{v})]^{2}} \sqrt{\left(\frac{1}{n}+\frac{1}{m}\right)} \text {, where } \hat{v}=\hat{\alpha} / \hat{\beta}
$$

where $Z_{(1-\alpha / 2)}$ is the $(1-\alpha / 2)^{\text {th }}$ percentile of the standard normal distribution.

\section{Simulation Study and Data Analysis}

\section{Simulation Study}

Results based on Monte Carlo simulations to compare the performance of the $R_{s, k}$ using different sample sizes are presented. 3,000 random sample of size 10(5)35 each from stress population, strength population were generated for $(\alpha, \beta)=$ $(3.0,1.0),(2.5,1.0),(2.0,1.0),(1.5,1.0),(1.0,1.0),(1.5,2.0),(1.5,2.5)$ and $(1.5,3.0)$ on lines of Bhattacharyya and Johnson (1974). The ML estimators of $\alpha$ and $\beta$ were then substituted in $v$ to obtain the reliability in a multicomponent stressstrength for $(s, k)=(1,3),(2,4)$. The average bias and average mean square error (MSE) of the reliability estimates over the 3,000 replications are given in Tables 1 and 2 . Average confidence length and coverage probability of the simulated $95 \%$ confidence intervals of $R_{s, k}$ are given in Tables 3 and 4 . The true value of reliability in multicomponent stress- strength with the given combinations of $(\alpha, \beta)$ for $(s, k)=(1,3)$ are $0.563,0.600,0.643,0.692,0.750,0.800,0.833,0.857$, 0.875 and for $(s, k)=(2,4)$ are $0.355,0.400,0.454,0.519,0.600,0.674,0.725$, 


\section{ESTIMATION OF RELIABILITY IN STRESS-STRENGTH}

$0.762,0.790$. Thus the true value of reliability in multicomponent stress- strength increases as $\beta$ increases for a fixed $\alpha$ whereas reliability in multicomponent stress- strength decreases as $\alpha$ increases for a fixed $\beta$ in both the cases of $(s, k)$. Therefore, the true value of reliability is increases as $v$ decreases and vice-versa. The average bias and average MSE are decreases as sample size increases for both $(s, k)$. It verifies the consistency property of the MLE of $R_{s, k}$. Also the bias is negative in both situations of $(s, k)$. Whereas, among the parameters the absolute bias and MSE are increases as $\alpha$ increases for a fixed $\beta$ in both the cases of ( $s$, $k$ ) and the absolute bias and MSE are decreases as $\beta$ increases for a fixed $\alpha$ in both the cases of $(s, k)$. The average length of the confidence interval also decreases as the sample size increases. The coverage probability is close to the nominal value in all cases but slightly less than 0.95 in most of the combinations. Overall, the performance of the confidence interval is good for all combinations of parameters. Whereas, among the parameters observed, the same phenomenon for average length and average coverage probability were observed in the case of average bias and MSE.

Table 1. Average bias of the simulated estimates of $R_{s, k}$

$(\alpha, \beta)$

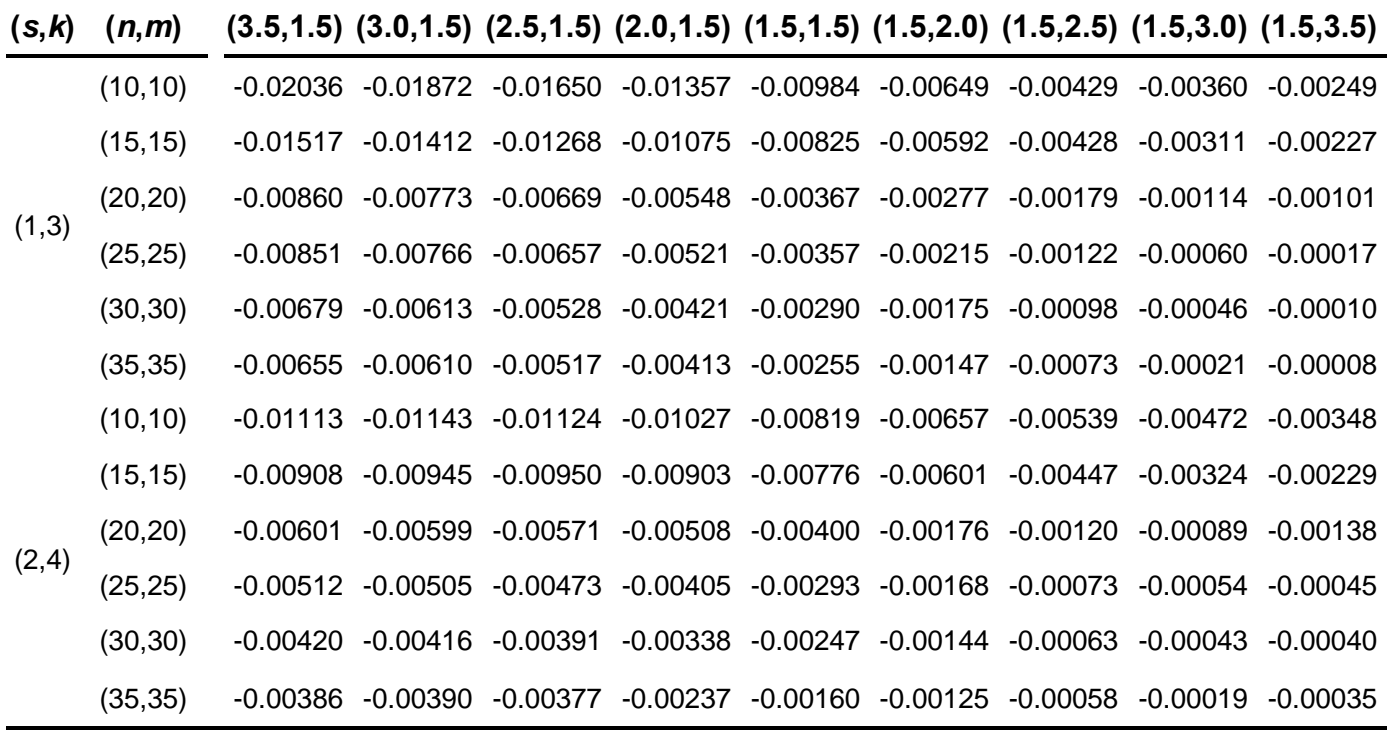




\section{GADDE SRINIVASA RAO}

Table 2. Average MSE of the simulated estimates of $R_{s, k}$

\begin{tabular}{|c|c|c|c|c|c|c|c|c|c|c|}
\hline$(s, k)$ & $(n, m$ & (3.5, & $(3.0$, & $(2.5$ & (2.0, & $\begin{array}{c}(\alpha, \beta) \\
(1.5,1.5)\end{array}$ & $(1.5$ & $(1.5$ & $(1.5$ & $(1.5,3$ \\
\hline \multirow{6}{*}{$(1,3$} & $(10,10)$ & 1529 & 0.01437 & 0.01300 & 0.01109 & 0.00854 & 0.00627 & 0.00477 & 0.00375 & 0.00303 \\
\hline & $(15,15)$ & 0.01052 & 0.00988 & 0.00894 & 0.00764 & 0.00590 & 0.00436 & 0.00334 & 0.00264 & 0.00214 \\
\hline & $(20,20)$ & 0.00713 & 0.00666 & 0.00599 & 0.00509 & 0.00392 & 0.00289 & 0.00221 & 0.00175 & 0.00143 \\
\hline & $(25,25)$ & 0.00592 & 0.00551 & 0.00494 & 0.00418 & 0.00322 & 0.00236 & 0.00181 & 0.00144 & 0.00117 \\
\hline & $(30,30)$ & 0.00460 & 0.00428 & 0.00383 & 0.00323 & 0.00248 & 0.00182 & 0.00139 & 0.00110 & 0.00090 \\
\hline & $(35,35)$ & 0.00402 & 0.00374 & 0.00337 & 0.00286 & 0.00220 & 0.00162 & 0.00125 & 0.00099 & 0.00081 \\
\hline \multirow{6}{*}{$(2,4)$} & $(10,10)$ & 0.01801 & 0.01877 & 0.01900 & 0.01831 & 0.01611 & 0.01320 & 0.01077 & 0.00889 & 0.00744 \\
\hline & $(15,15)$ & 0.01285 & 0.01337 & 0.01351 & 0.01298 & 0.01140 & 0.00932 & 0.00762 & 0.00630 & 0.00529 \\
\hline & $(20,20)$ & 0.00906 & 0.00936 & 0.00938 & 0.00895 & 0.00781 & 0.00635 & 0.00518 & 0.00429 & 0.00361 \\
\hline & $(25,25)$ & 0.00754 & 0.00778 & 0.00778 & 0.00740 & 0.00643 & 0.00522 & 0.00426 & 0.00352 & 0.00296 \\
\hline & $(30,30)$ & 0.00594 & 0.00612 & 0.00610 & 0.00578 & 0.00500 & 0.00404 & 0.00328 & 0.00271 & 0.00228 \\
\hline & $(35,35)$ & 0.00522 & 0.00538 & 0.00538 & 0.00512 & 0.00445 & 0.00361 & 0.00295 & 0.00244 & 0.00205 \\
\hline
\end{tabular}

Table 3. Average confidence length of the simulated 95\% confidence intervals of $R_{s, k}$

$$
(\alpha, \beta)
$$

\begin{tabular}{|c|c|c|c|c|c|c|c|c|c|c|}
\hline$;, k)$ & $(n, m)$ & 5 & 1.5 & & 01 & 1 & 5. & 5 & 5 & \\
\hline \multirow{6}{*}{$(1,3)$} & $(10,10)$ & 0.4091 & 0.4021 & 0.3880 & 0.3632 & 0.3224 & 0.2763 & 0.2400 & 0.2113 & 0.1884 \\
\hline & $(15,15)$ & 0.3399 & 0.3334 & 0.3210 & 0.2999 & 0.2658 & 0.2279 & 0.1981 & 0.1747 & 0.1559 \\
\hline & $(20,20)$ & 0.2975 & 0.2911 & 0.2794 & 0.2601 & 0.2296 & 0.1961 & 0.1701 & 0.1497 & 0.1335 \\
\hline & $(25,25)$ & 0.2675 & 0.2617 & 0.2512 & 0.2338 & 0.2063 & 0.1762 & 0.1529 & 0.1346 & 0.1201 \\
\hline & $(30,30)$ & 0.2453 & 0.2398 & 0.2300 & 0.2140 & 0.1887 & 0.1612 & 0.1398 & 0.1232 & 0.1099 \\
\hline & $(35,35)$ & 0.2277 & 0.2226 & 0.2135 & 0.1986 & 0.1753 & 0.1498 & 0.1301 & 0.1146 & 0.1023 \\
\hline \multirow{6}{*}{$(2,4)$} & $(10,10)$ & 0.4569 & 0.4719 & 0.4802 & 0.4760 & 0.4498 & 0.4055 & 0.3637 & 0.3274 & 0.2966 \\
\hline & $(15,15)$ & 0.3834 & 0.3953 & 0.4012 & 0.3968 & 0.3739 & 0.3366 & 0.3019 & 0.2719 & 0.2466 \\
\hline & $(20,20)$ & 0.3396 & 0.3492 & 0.3533 & 0.3479 & 0.3260 & 0.2920 & 0.2610 & 0.2346 & 0.2123 \\
\hline & $(25,25)$ & 0.3056 & 0.3143 & 0.3180 & 0.3131 & 0.2934 & 0.2628 & 0.2350 & 0.2112 & 0.1912 \\
\hline & $(30,30)$ & 0.2813 & 0.2891 & 0.2923 & 0.2875 & 0.2691 & 0.2409 & 0.2153 & 0.1935 & 0.1752 \\
\hline & $(35,35)$ & 0.2611 & 0.2684 & 0.2714 & 0.2670 & 0.2500 & 0.2240 & 0.2003 & 0.1801 & 0.1631 \\
\hline
\end{tabular}




\section{ESTIMATION OF RELIABILITY IN STRESS-STRENGTH}

Table 4. Average coverage probability of the simulated 95\% confidence intervals of $R_{s, k}$

\begin{tabular}{|c|c|c|c|c|c|c|c|c|c|c|}
\hline$(s, l$ & $(n, m)$ & 5, & ( & ( & $(2$ & $(1.5,1.5)$ & & & & \\
\hline \multirow{6}{*}{$(1,3)$} & $(10,10)$ & 0.9090 & 0.9140 & 0.9193 & 0.9280 & 0.9317 & 0.9303 & 0.9267 & 0.9260 & 0.9267 \\
\hline & $(15,15)$ & 0.9120 & 0.9150 & 0.9187 & 0.9213 & 0.9250 & 0.9273 & 0.9290 & 0.9287 & 0.9253 \\
\hline & $(20,20)$ & 0.9303 & 0.9347 & 0.9370 & 0.9390 & 0.9390 & 0.9377 & 0.9380 & 0.9347 & 0.9303 \\
\hline & $(25,25)$ & 0.9227 & 0.9267 & 0.9317 & 0.9360 & 0.9383 & 0.9373 & 0.9357 & 0.9333 & 0.9277 \\
\hline & $(30,30)$ & 0.9353 & 0.9403 & 0.9423 & 0.9463 & 0.9490 & 0.9463 & 0.9433 & 0.9400 & 0.9390 \\
\hline & $(35,35)$ & 0.9317 & 0.9330 & 0.9353 & 0.9387 & 0.9387 & 0.9400 & 0.9363 & 0.9337 & 0.9297 \\
\hline \multirow{6}{*}{$(2,4)$} & $(10,10)$ & 0.9113 & 0.9153 & 0.9203 & 0.9243 & 0.9273 & 0.9277 & 0.9257 & 0.9250 & 0.9243 \\
\hline & $(15,15)$ & 0.9103 & 0.9160 & 0.9190 & 0.9193 & 0.9233 & 0.9263 & 0.9297 & 0.9260 & 0.9223 \\
\hline & $(20,20)$ & 0.9310 & 0.9340 & 0.9370 & 0.9380 & 0.9367 & 0.9367 & 0.9360 & 0.9323 & 0.9287 \\
\hline & $(25,25)$ & 0.9237 & 0.9287 & 0.9313 & 0.9353 & 0.9370 & 0.9350 & 0.9323 & 0.9307 & 0.9257 \\
\hline & $(30,30)$ & 0.9357 & 0.9397 & 0.9437 & 0.9437 & 0.9477 & 0.9457 & 0.9420 & 0.9403 & 0.9397 \\
\hline & $(35,35)$ & 0.9297 & 0.9317 & 0.9367 & 0.9360 & 0.9407 & 0.9393 & 0.9367 & 0.9327 & 0.9300 \\
\hline
\end{tabular}

\section{Data Analysis}

Strength data, which was originally reported by Badar and Priest (1982), represents the strength measured in GPA for single carbon fibers and impregnated 1,000-carbon fiber tows. Single fibers were tested under tension at gauge lengths of $20 \mathrm{~mm}$ (Data Set I) and $10 \mathrm{~mm}$ (Data Set II), with sample sizes $n=69$ and $m=$ 63 respectively (see Data sets I and II).

Data Set I (gauge lengths of $20 \mathrm{~mm}$ ).

$1.312,1.314,1.479,1.552,1.700,1.803,1.861,1.865,1.944,1.958,1.966,1.997,2.006$, $2.021,2.027,2.055,2.063,2.098,2.140,2.179,2.224,2.240,2.253,2.270,2.272,2.274$, 2.301, 2.301, 2.359, 2.382, 2.382, 2.426, 2.434, 2.435, 2.478, 2.490, 2.511, 2.514, 2.535, $2.554,2.566,2.570,2.586,2.629,2.633,2.642,2.648,2.684,2.697,2.726,2.770,2.773$, $2.800,2.809,2.818,2.821,2.848,2.880,2.809,2.818,2.821,2.848,2.880,2.954,3.012$, $3.067,3.084,3.090,3.096,3.128,3.233,3.433,3.585,3.585$.

Data Set II (gauge lengths of $10 \mathrm{~mm}$ ).

$1.901,2.132,2.203,2.228,2.257,2.350,2.361,2.396,2.397,2.445,2.454,2.474,2.518$, $2.522,2.525,2.532,2.575,2.614,2.616,2.618,2.624,2.659,2.675,2.738,2.740,2.856$, $2.917,2.928,2.937,2.937,2.977,2.996,3.030,3.125,3.139,3.145,3.220,3.223,3.235$, $3.243,3.264,3.272,3.294,3.332,3.346,3.377,3.408,3.435,3.493,3.501,3.537,3.554$, $3.562,3.628,3.852,3.871,3.886,3.971,4.024,4.027,4.225,4.395,5.020$. 


\section{GADDE SRINIVASA RAO}

Surles and Padgett $(1998,2001)$ observed that generalized Rayleigh works well for strength data. Raqab and Kundu (2005) analyzed the data by subtracting 1.0 and 1.8 from the first and second data set respectively. The transformed data sets correspond to $20 \mathrm{~mm}$ and $10 \mathrm{~mm}$ gauge lengths are assumed to follow $G R(\alpha, \lambda)$ and $G R(\beta, \lambda)$ respectively. The obtained final estimates for these two data sets are $\hat{\alpha}=2.4421, \hat{\beta}=1.4216$, and $\hat{\lambda}=0.8598$. Also they checked the validity of the models using the Kolmogorov-Smirnov (K-S) tests for each data set. It was observed that for Data Sets I and II, the K-S distances are 0.09 and 0.12 with the corresponding $p$ values of 0.6069 and 0.2845 respectively. It indicates that the GR model provides reasonable fit to the transformed data sets.

Based on estimates of $\alpha$ and $\beta$ the MLE of $R_{s, k}$ become $\hat{R}_{1,3}=0.63588$ and $\hat{R}_{2,4}=0.44484$. The $95 \%$ confidence intervals for $R_{1,3}$ become $(0.55680,0.71496)$ and for $R_{2,4}$ become $(0.34387,0.54581)$.

\section{Conclusions}

This article used real data sets to investigate multicomponent stress-strength reliability for a generalized Rayleigh distribution when both stress, strength variates follow the same population. Asymptotic confidence intervals for multicomponent stress-strength reliability were estimated using the ML method. Simulation results indicate that the average bias and average MSE decreases as sample size increases in both cases of $(s, k)$. Among the parameters the absolute bias and MSE are increases (decreases) as $\alpha$ increases ( $\beta$ increases) in both the cases of $(s, k)$. The length of the confidence interval also decreases as the sample size increases and coverage probability is close to the nominal value in all sets of parameters considered.

\section{References}

Awad, M. \& Gharraf, K. (1986). Estimation of $p(Y<X)$ in Burr case: A comparative study. Communications in Statistics - Simulations \& Comp., 15: 389403.

Badar, M. G. \& Priest, A. M. (1982). Statistical aspects of fiber and bundle strength in hybrid composites. In T. Hayashi, K. Kawata, and S. Umekawa (eds.), Progress in Science and Engineering Composites, (pp. 1129-1136). Tokyo: ICCM-IV. 


\section{ESTIMATION OF RELIABILITY IN STRESS-STRENGTH}

Bhattacharyya, G. K. \& Johnson, R. A. (1974). Estimation of reliability in multicomponent stress - strength model. JASA, 69: 966-970.

David, H. A. (1981). Order Statistics. New York: John Wiley and Sons.

Downtown, F. (1973). The estimation of $p(X>Y)$ in the normal case. Technometrics, 15: 551-558.

Enis, P. \& Geisser, S. (1971). Estimation of the probability that $Y<X . J A S A$, 66: $162-168$.

Kendall, M. G. \& Stuart, A. (1979). The Advanced Theory of Statistics, (Vol. 2). London: Charles Griffin and Company Limited.

Kundu, D. \& Gupta, R. D. (2005). Estimation of $p(Y<X)$ for the generalized exponential distribution. Metrika, 61(3): 291-308.

Kundu, D. \& Gupta, R. D. (2006). Estimation of $p(Y<X)$ for Weibull distribution, IEEE Transactions on Reliability, 55(2): 270-280.

Kundu, D. \& Raqab, M. Z. (2005). Generalized Rayleigh distribution: different methods of estimation. Computational Statistics and Data Analysis, 49: $187-200$.

Kundu, D. and Raqab, M. Z. (2009). Estimation of $\mathrm{R}=p(Y<X)$ for threeparameter Weibull distribution, Statistics and Probability Letters, 79: 1839-1846.

McCool, J. I. (1991). Inference on $p(Y<X)$ in the Weibull case, Communications in Statistics - Simulations \& Comp., 20: 129-148.

Mudholkar, G. S. \& Srivastava, D. K. (1993). Exponentiated Weibull family for analyzing bathtub failure data, IEEE Transactions on Reliability, 42: 299-302.

Nandi, S. B. \& Aich, A. B. (1994). A note on estimation of $p(X>Y)$ for some distributions useful in life- testing, IAPQR Transactions, 19(1): 35 - 44.

Pandey, M. \& Uddin, B. (1985). Estimation of reliability in multicomponent stress - strength model following Burr distribution. Proceedings of the First Asian congress on Quality and Reliability, (pp. 307 - 312). New Delhi, India.

Rao, C. R. (1973). Linear Statistical Inference and its Applications. India: Wiley Eastern Limited.

Raqab, M. Z. \& Kundu, D. (2005). Comparison of different estimators of $p(Y<X)$ for a scaled Burr type $\mathrm{X}$ distribution, Communications in Statistics Simulation and Computation, 34(2): 465 - 483.

Raqab, M. Z., Madi, M. T. \& Kundu, D. (2008). Estimation of $p(Y<X)$ for the 3-parameter generalized exponential distribution, Communications in Statistics - Theory and Methods, 37(18): 2854 - 2864. 


\section{GADDE SRINIVASA RAO}

Srinivasa Rao, G. \& Kantam, R. R. L. (2010). Estimation of reliability in multicomponent stress-strength model: log-logistic distribution, Electronic

Journal of Applied Statistical Analysis, 3(2): 75-84.

Surles, J. G. \& Padgett, W. J. (1998). Inference for $p(Y<X)$ in the Burr Type X model, Journal of Applied Statistical Sciences, 7: 225 - 238.

Surles, J. G. \& Padgett, W. J. (2001). Inference for reliability and stressstrength for a scaled Burr Type X distribution, Lifetime Data Analysis, 7: 187-200. 